\title{
Age-related changes in trabecular bone microstructures Global and local morphometry
}

\author{
Journal Article \\ Author(s): \\ Stauber, M.; Müller, Ralph (iD) \\ Publication date: \\ 2006-04 \\ Permanent link: \\ https://doi.org/10.3929/ethz-b-000025295 \\ Rights / license: \\ In Copyright - Non-Commercial Use Permitted \\ Originally published in: \\ Osteoporosis International 17(4), https://doi.org/10.1007/s00198-005-0025-6
}




\title{
Age-related changes in trabecular bone microstructures: global and local morphometry
}

\author{
M. Stauber $\cdot$ R. Müller
}

Received: 6 April 2005 / Accepted: 29 September 2005 / Published online: 31 December 2005

(C) International Osteoporosis Foundation and National Osteoporosis Foundation 2005

\begin{abstract}
Introduction: A recently developed method allows investigating trabecular bone on an elemental (rod/plate) level. With this method, it is possible to measure local morphometric parameters such as thickness or orientation directly on the extracted rods and plates. Age-related changes of trabecular microarchitecture can thus be investigated on an elemental level, which may help to improve the understanding of age-related bone failure mechanism as well as the effect of pharmaceutical intervention in the prevention of such fractures. Methods: Autopsies from femoral heads (FH) and lumbar spine (LS) were analyzed by global morphometry. Additionally, the trabecular structures were decomposed into rods and plates for the analysis with local morphometry. These morphometric indices were related to age using an analysis of covariance to test for gender differences and linearity with age. Results: In this study, age-related changes showed no gender but site differences. In LS, rods were thinned in aging and finally vanish from the structure, causing a transformation of the trabecular bone structure to longer and, on average, thicker rods. In FH, changes were expressed by a simultaneous thinning and loss of interconnecting trabeculae and perforation of plates leading to new plates and rods. Results were mostly in agreement with earlier findings using descriptive analysis of the aging process. Conclusion: Here we present for the first time preliminary quantitative evidence of changes in the local microstructure, i.e., individual rods and plates. Nevertheless, the number of samples was too small to make for ready conclusions. We conclude that the combination of local and global morphometry is a useful method for a detailed and quantitative description of age-related changes in bone microstructure.
\end{abstract}

M. Stauber · R. Müller $(\bowtie)$

Institute for Biomedical Engineering,

Swiss Federal Institute of Technology (ETH)

and University of Zürich,

Moussonstrasse 18,

8044 Zürich, Switzerland

e-mail: ralph.mueller@ethz.ch

Tel.: +41-44-6324592

Fax: +41-44-6321214
Keywords Age-related changes - Local morphometry · Osteoporosis - Trabecular bone - Trabecular plates .

Trabecular rods

\section{Introduction}

The definition of osteoporosis changed over the years with increasing knowledge of this disease. Where earlier, osteoporosis was defined as an absolute decrease in the amount of bone [1-3] to a level below that required for mechanical support [4], later definitions added the importance of microarchitecture [5, 6] and bone quality [7], a term referring to architecture, turnover, damage accumulation, and mineralization. With the changed definition of osteoporosis, the focus of investigation also changed over the years. Most early studies in age-related bone changes concentrated in the description of the amount of bone volume [1-3, 8-17], and only few investigated structural parameters such as bone surface density (BS/TV) $[11,14$, 15], trabecular number (Tb.N) [14, 18], trabecular thickness (Tb.Th) [12, 14], or trabecular spacing (Tb.Sp) [12]. This was based on the intuitive idea that the mechanical properties of trabecular bone depend strongly on its apparent density or bone volume $[19,20]$, and it became an indisputable fact that bone is lost in aging people. However, Hui et al. demonstrated that even for constant bone mass, fracture risk increases with age [21]. Thus, bone quality changes with age, independent of bone mass, and components such as microarchitecture, turnover, damage accumulation, and mineralization must be taken into account in determining bone strength.

Parfitt et al. were first to highlight the evidence of trabecular bone microstructure in the etiology of osteoporosis and bone fractures [22]. With their newly introduced parallel-plate model, they investigated architectural changes with age, such as spacing and thickness of trabecular plate structures, and found that the reduction in bone volume was mainly due to a reduction in plate density, with no significant decrease in plate thickness $[22,23]$. The authors proposed that the process of plate removal was initiated by 
an excessive depth of osteoclastic resorption cavities, leading to focal perforation of plates followed by progressive enlargement of the perforation with conversion of plates to rods. It was also proposed that a potential thinning of residual elements could arise from incomplete refilling by osteoblasts of resorption cavities of normal or reduced size [24]. This theory stimulated research in trabecular bone structure related to aging, and different methods were used to investigate the thickness and spacing of trabecular elements [25-30].

With the improvement in spatial resolution of threedimensional imaging systems such as microcomputed tomography $(\mu \mathrm{CT})[31]$, peripheral quantitative-computed tomography (pQCT) $[32,33]$, and magnetic resonance imaging (MRI) [34-36], it became possible to assess trabecular bone microstructure in three-dimensional space. This pushed the research field toward the assessment of new, unbiased, three-dimensional (3-D) quantities such as the model-independent assessment of mean $\mathrm{Tb}$.Th and mean trabecular separation (spacing) (Tb.Sp) [37], structure model index (SMI) [38], mean curvature of trabecular bone structure $(<\mathrm{H}>)$ [39], or connectivity density (Conn. D) [40]. All these parameters were also related to changes with age and diseases [41-43].

Recently, we proposed a new method for the local morphometric analysis of single trabecular rod and plate elements [44-46]. This new method allows computing morphometric parameters, such as volume, surface, thickness, and orientation, directly on a trabecular level (i.e., individual rods and plates) and it is possible, for the first time, to verify in 3-D space whether the thickness of horizontal and vertical rods are age dependent. Local morphometry also opens the doors to the investigation of new parameters, such as the slenderness of rods, which is important in determining whether buckling is a potential failure mode. To complete the picture in age-related changes of trabecular bone, we also included global morphometric parameters such as bone volume density (BV/TV) and Tb. $\mathrm{Sp}$, which are better measured by means of global than local methods. The overall aim of this study was to achieve a better understanding of age-related changes of trabecular bone microstructure.

\section{Methods}

In this study, we analyzed human trabecular bone samples that were measured earlier as part of the European Union BIOMED I Concerted Action "Assessment of Bone Quality in Osteoporosis" [47, 48]. The analyzed data set encompasses 131 samples harvested from 70 donors at two different anatomical sites; femoral head (FH) and second lumbar spine (LS). These two sites were shown to have similar lifetime risk for fracture, which was about three times higher in women than in men $[49,50]$. Bone samples were recruited from subjects in the postmortem room in standardized conditions from subjects of all ages and different pathological conditions. The main causes of death were heart failure, pneumonia, stroke, and sepsis. The samples were extracted along their site-specific loading axis. Detailed clinical donor characteristics included in this BIOMED I project have been described elsewhere [51, 52]. No donors were excluded due to known skeletal diseases, metabolic diseases, or drugs known to affect the skeleton. In that sense, the study population was not considered a normal population with respect to their bone status but a typical cross-section of the hospital population at the time of the study. The numbers of samples and mean ages per site and gender are shown in Table 1.

All samples were scanned using a microcomputed tomography $(\mu \mathrm{CT})$ system $(\mu \mathrm{CT} 20$, Scanco Medical AG, Switzerland) providing a spatial resolution of $28 \mu \mathrm{m}$. This system and scanning procedure is described elsewhere in detail [31], and the data set has previously been used for several investigations $[46,47,53]$. A 4-mm cubic region of interest (TV) was selected from all samples. Using Gaussian filtration and global thresholding [47], binary images were created presenting either bone or background. A component-labeling algorithm was applied to the binary images to remove all parts not connected to the main structure.

These images were then used for both global and local morphometry. For global morphometry, standard 3-D algorithms were used to compute bone volume density (BV/TV), bone surface density (BS/TV), and specific bone surface (BS/BV), as well as Tb.Sp, Tb.N, and Tb.Th. These algorithms are described in detail elsewhere [37, 47]. Furthermore, we also computed the $<\mathrm{H}>$ [39] also denoted to as trabecular bone pattern factor (TBPf) [54], and the SMI [38]. All these parameters were computed in 3-D space without using any model assumptions and are summarized in Fig. 2 and Table 2.

For local morphometry, the component-labeled images were first spatially decomposed into rods and plates. A detailed description of the volumetric spatial decomposition algorithm as well as local morphometry can be found elsewhere [46] where here only a short description of the main procedures is provided. First, the component-labeled images were skeletonized using a 3-D homotopic, isotropic, and shape-preserving thinning algorithm [55, 56], yielding in a two-voxel thick, symmetric skeleton. Subsequently, a topology-preserving algorithm called conditional erosion was used to reduce this skeleton to 1 voxel thick-

Table 1 Number and age ranges of the trabecular bone samples per gender and site

\begin{tabular}{|c|c|c|c|c|}
\hline \multirow[t]{2}{*}{ Site } & \multicolumn{2}{|l|}{ Female } & \multicolumn{2}{|l|}{ Male } \\
\hline & $\begin{array}{l}\text { Femoral } \\
\text { head }\end{array}$ & $\begin{array}{l}\text { Lumbar } \\
\text { spine }\end{array}$ & $\begin{array}{l}\text { Femoral } \\
\text { head }\end{array}$ & $\begin{array}{l}\text { Lumbar } \\
\text { spine }\end{array}$ \\
\hline Number & 28 & 29 & 36 & 38 \\
\hline Mean age & 71.1 & 72.1 & 67.0 & 66.7 \\
\hline $\mathrm{SD}$ & 15.6 & 15.7 & 14.9 & 15.2 \\
\hline Minimum & 23 & 23 & 28 & 28 \\
\hline Maximum & 92 & 92 & 87 & 87 \\
\hline$p$ value* & 0.29 & 0.16 & & \\
\hline
\end{tabular}

*Site-wise students t-test for female vs male 
Table $2 P$ values of the analysis of covariance for the global morphometric indices

\begin{tabular}{llrlll}
\hline Index & Site & \multicolumn{1}{c}{ Age $^{\mathrm{a}}$} & Gender & Age:gender $^{\mathrm{b}}$ & Change $^{\mathrm{c}}$ \\
\hline $\mathrm{BV} / \mathrm{TV}$ & FH & 0.065 & 0.132 & 0.770 & - \\
& LS & $\mathbf{0 . 0 0 4}$ & 0.437 & 0.505 & -6.4 \\
$\mathrm{BS} / \mathrm{TV}$ & FH & 0.187 & 0.194 & 0.897 & - \\
& LS & $<\mathbf{0 . 0 0 1}$ & 0.356 & 0.399 & -6.8 \\
$\mathrm{BS} / \mathrm{BV}$ & FH & 0.112 & 0.263 & 0.533 & - \\
& LS & 0.128 & 0.743 & 0.494 & - \\
$\mathrm{Tb} . \mathrm{Sp}$ & FH & 0.157 & 0.329 & 0.779 & - \\
& LS & $\mathbf{0 . 0 0 8}$ & 0.506 & 0.687 & 4.9 \\
$\mathrm{~Tb} . \mathrm{N}$ & FH & 0.123 & 0.177 & 0.896 & - \\
& LS & $\mathbf{0 . 0 0 3}$ & 0.656 & 0.856 & -3.6 \\
$\mathrm{~Tb} . \mathrm{Th}$ & FH & 0.201 & 0.531 & 0.584 & - \\
& LS & 0.480 & 0.637 & 0.896 & - \\
$<\mathrm{H}>$ & FH & 0.077 & 0.052 & 0.837 & - \\
& LS & 0.463 & 0.668 & 0.229 & - \\
$\mathrm{SMI}$ & FH & 0.072 & 0.070 & 0.528 & - \\
& LS & $\mathbf{0 . 0 1 2}$ & 0.268 & 0.357 & 6.0 \\
\hline
\end{tabular}

$B V / T V$ bone volume density, $B S / T V$ bone surface density, $B S / B V$ specific bone surface, Tb.Sp trabecular separation, $T b . N$ trabecular number, $T b . T h$ trabecular thickness, $\langle H\rangle$ mean curvature of trabecular bone structure, $S M I$ structure model index, $F H$ femoral head, $L S$ lumbar spine

${ }^{a}$ Bold numbers are smaller than 0.05

${ }^{\mathrm{b}}$ Interaction term age:gender

${ }^{\mathrm{c}}$ Change per decade in percent

ness [46]. These skeletons are a very rough, 1-voxel-thick representation of the original bone structure where rods with elliptic cross-section may be represented as slender plates. To overcome this problem, we applied an optimization algorithm to the skeleton to get a reasonable representation of rods and plates and to reduce surface noise. The slenderness parameter used for this optimization step was set to 10 , which was visually yielding in reasonable results [46]. The optimized skeleton was then characterized by a slightly modified point-classification algorithm originally devised by Saha et al. [57], which was able to compute for each voxel within the skeleton whether it was a surface point, a surface end point, an arc point, an arc end point, an arc-arc intersection point, an arc-surface intersection point, a surface-surface intersection point, or an isolated point. This classified skeleton was spatially decomposed into rods and plates, which were expanded to their original size by applying a so called multicolor dilation algorithm [46]. This operation resulted in a spatially decomposed trabecular bone structure where all rods and plates were labeled with an individual number (Fig. 1a,c).

Local morphometry was then used to compute elementbased indices [46]. These indices were computed and averaged for rods and plates separately (Fig. 1b,d). The length of the rods $(<$ Ro.Le $>)$ was determined directly from the point-classified skeleton by computing the distance of the two nodes. This operation was applied only to rods since the orientation of plates is not clearly defined. The orientation of the rods $(<\operatorname{Ro} . \theta>)$ was defined as the angle spanned by the line connecting the two nodes and the Zaxis of the image. Since all samples were extracted along their site-specific loading axis, the Z-axis was collinear with the loading axis, and thus, the orientation represents the orientation as compared with the loading axis. We used this angle to group vertical elements $\left(\theta<=45^{\circ}\right)$ and horizontal elements $\left(\theta>45^{\circ}\right)$. All other parameters such as the thickness or volume of the elements were determined by applying standard morphometry algorithms to the rods and plates. In this paper, we denote $<$ Ro.Th $>$ and $<$ Pl.Th $>$ to be the mean thickness of all rods and plates, respectively, in a structure. The mean thickness of all vertical and horizontal rods are denoted to as $\left\langle\mathrm{Ro}_{\mathrm{V}}\right.$. Th $>$ and $\left\langle\mathrm{Ro}_{\mathrm{H}}\right.$. Th $>$, respectively. The mean slenderness of the rods, as computed from the length over the thickness, is denoted to $<$ Ro.Sl $>$. Furthermore, we computed the plate volume density (Pl.BV/BV), which is defined as total plate volume divided by total volume of interest in percent. This parameter was shown to represent structure type similar to the globally determined SMI but in a more direct, unambiguous way [46].

For the statistical analysis, the GNU statistical computation and graphics package R (Version 2.0.1; http://www.r-project. org) was used. We conducted an analysis of covariance to determine whether the morphometric indices had a linear trend with age and to find differences between women and men. For the indices that showed an age-related linear trend, we used the linear model to express these changes. The linear regression was expressed by arbitrarily setting the value at 30 years (young adult) to $100 \%$, which allowed us to express the increase/decrease of a certain parameter in percentage per decade relative to this age. With this, the regression line is clearly defined, and the $100 \%$ level, which was arbitrarily chosen at the age of 30 , could easily be moved to any other age. This analysis of covariance was performed site wise, and the resulting $p$ values are shown in Tables 2 and 3 for global and local morphometry, respectively. The data was visualized by scatter plots, and LOWESS fits [58, 59] were used to highlight trends (Figs. 2, 3 and 4). The same statistical analysis was performed for all samples of donors aged 59 years and older.

\section{Results}

Generally, we can say that for all morphometric indices, the variation between subjects in any given site was large as compared with age-related changes. For this reason, it was difficult to formulate general laws, and for most parameters, it was not so clear whether the relation with age was linear. Nevertheless, the data gives important indications and trends in bone changes with increasing age. The levels and trends of most morphometric indices were different for the three sites.

The analysis of covariance revealed no differences between female and male bone samples (Tables 2 and 3). Therefore, male and female data was pooled for further analysis and displayed in the same scatter plots (Figs. 2, 3, and 4). 
Fig. 1 Spatial decomposition of trabecular bone structures into rods and plates. The images on the left-hand side show the extracted elements after the spatial decomposition by different colors, the images on the right-hand side show which elements were identified as rods (blue) and plates (red), respectively. The three structures are from femoral head $(\mathbf{a}, \mathbf{b})$ and lumbar spine (c, d) to represent a platelike and rodlike trabecular bone structure
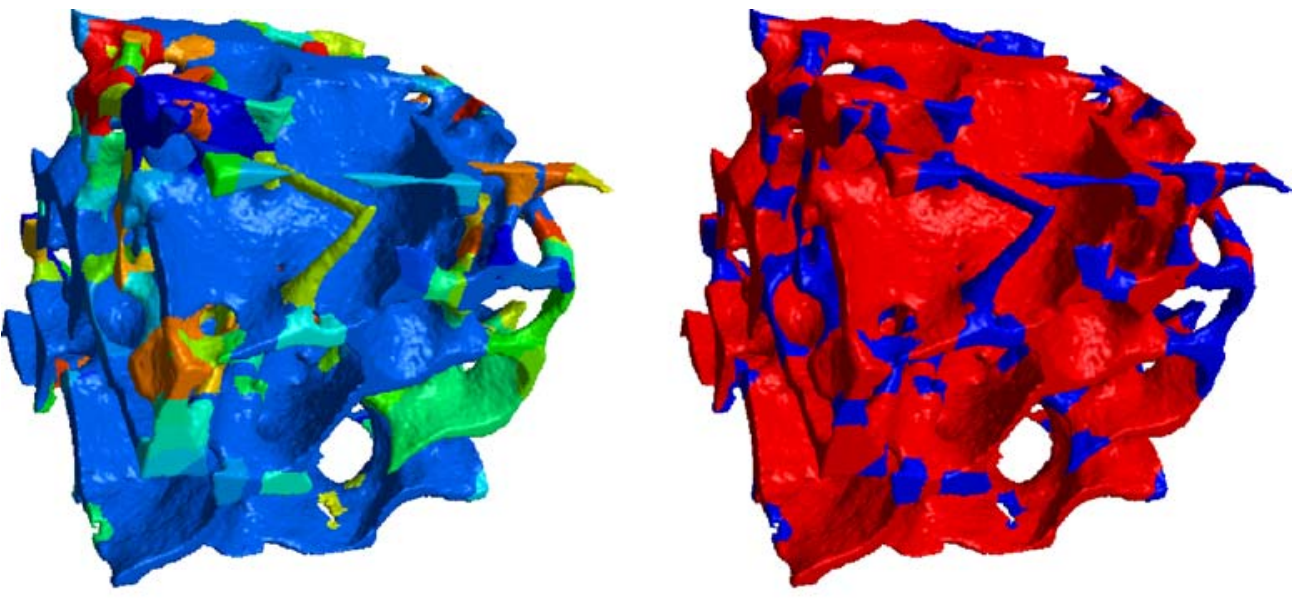

A

B

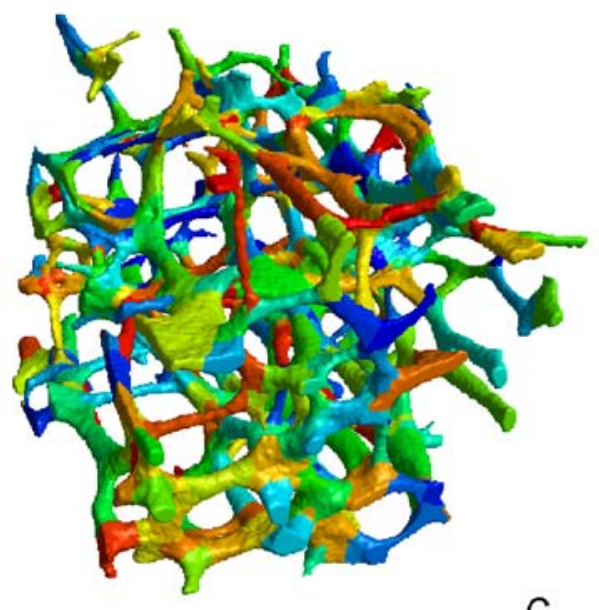

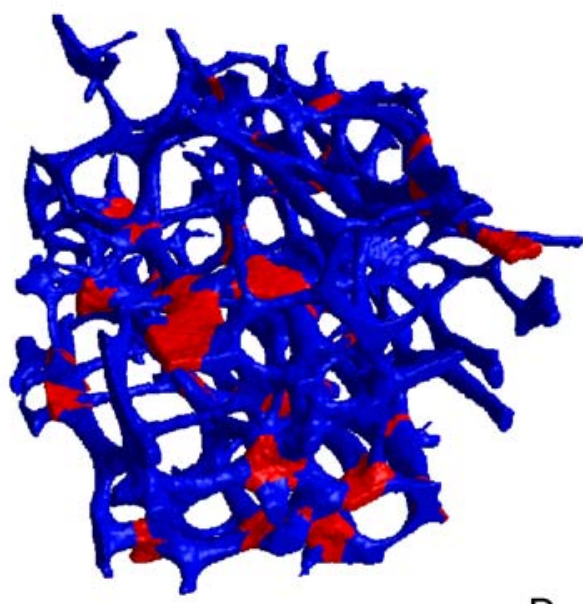

D
From global morphometry, we found an age-related decrease in $\mathrm{BV} / \mathrm{TV}$, which showed a linear relation in LS that was found to be $6.4 \%$ per decade. However, the variation was large at all ages. The sites differed in BV/TV, being high for $\mathrm{FH}$ and low for LS. A similar pattern was found for $\mathrm{BS} / \mathrm{TV}$, where a linear relation with age was found in LS. This parameter seemed to decrease more dramatically at higher ages ( $>70$ years) similarly for both sites. $\mathrm{BS} / \mathrm{BV}$, on the other hand, showed no age-related changes for any of the sites. This parameter was large in LS and low in $\mathrm{FH}$, which can be explained by the different sizes of the structure elements. There was a general increase in Tb.Sp, which showed a linear relation with age in LS. Again, the increase was more pronounced after the age of about 70 years. In LS, spacing was found to be increased by $4.9 \%$ per decade. Spacing was large in LS and small in FH. Accordingly, Tb.N decreased with age and was large in FH and small in LS. This parameter also showed a linear relation to age in LS. No significant change with age was found for the globally determined Tb.Th and $<\mathrm{H}>$. The SMI increased slightly in LS only. These results are summarized in Fig. 2 and Table 2.
From local morphometry, we found an age-related decrease in the number of rods in LS. On average, a 90year-old person would have a 50\% reduced number in rods as compared with a 30 -year-old subject. The other site showed no obvious trend in this parameter. The ratio of the number of rods to the number of plates as well as $\mathrm{Pl} . \mathrm{BV} / \mathrm{BV}$ showed no age-related trend, and the levels in Pl.BV/BV were quite different for the two sites. The $<$ Ro.Sl $>$ increased with age and showed a linear relation in FH and LS. The $<$ Ro.Th $>$ and $<$ Pl.Th $>$ showed a trend only in LS, where an age-related increase was observed. The increase was observed in both $<\mathrm{Ro}_{\mathrm{V}}$. Th $>$ and $<\mathrm{Ro}_{\mathrm{H}}$. Th $>$ elements. With increasing age, rods tended to be oriented along the anatomical axis ( $\mathrm{Z}$-axis), which is reflected in LS by a linear increase of the percentage of vertical rods $(\mathrm{Nr}$. Rods $_{\mathrm{V}} / \mathrm{Nr}$.Rods). These results are summarized in Figs. 3 and 4 and Table 3. By performing the same statistical analysis including only the samples aged 59 years and older, all $p$-values became insignificant. The variation at these ages was obviously too large and the observed time interval too short to reveal significant linear trends over time. 

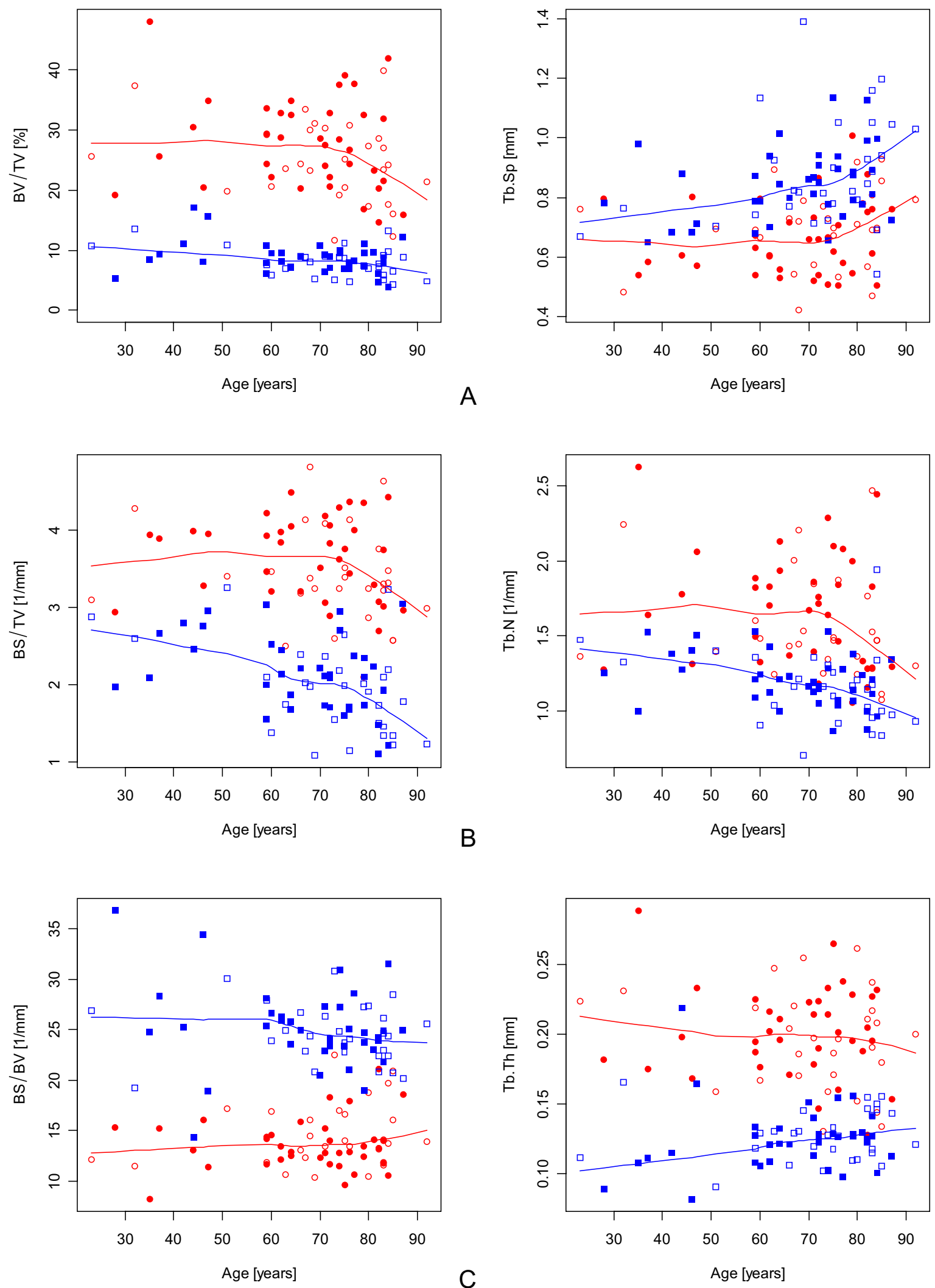

Fig. 2 Scatter plots with LOWESS fits for the global morphometric indices. The measured indices are bone volume density (BV/TV), bone surface density (BS/TV), specific bone surface (BS/BV), trabecular spacing (Tb.Sp), trabecular number (Tb.N), and trabec-

ular thickness (Tb.Th). Open symbols denote female, and closed symbols denote male, where data from femoral head are displayed as circles $(\circ, \bullet)$ and data from lumbar spine as squares $(\square, \boldsymbol{\square})$ 

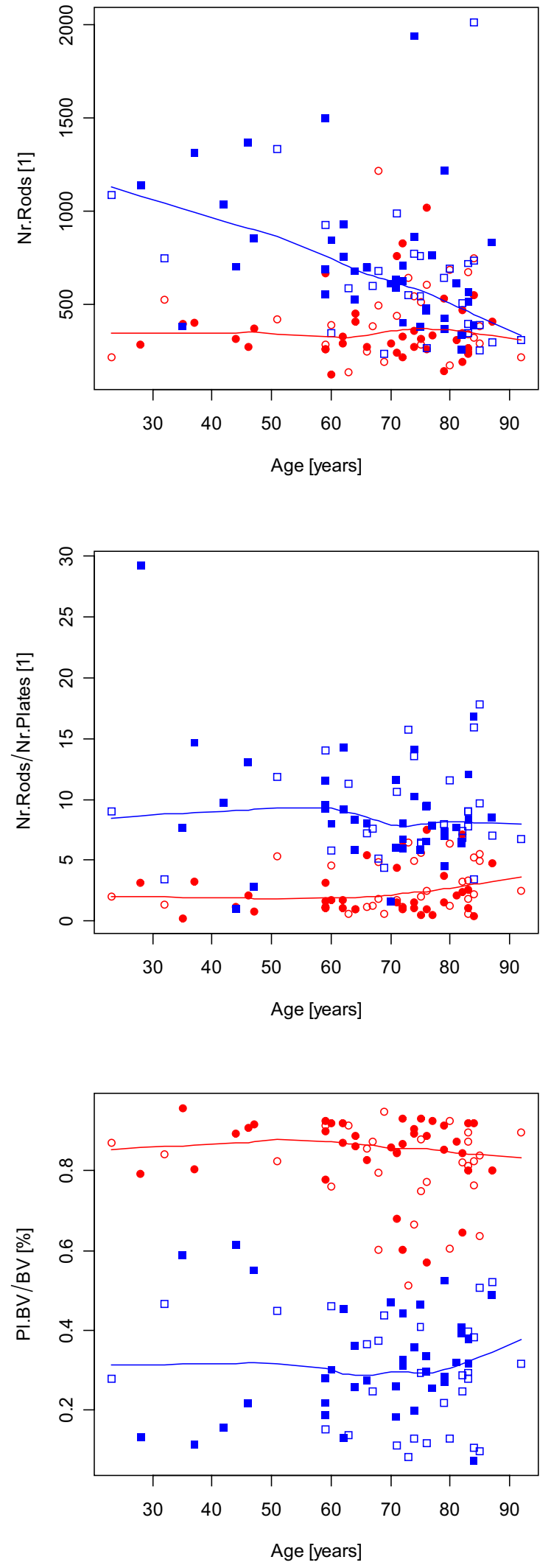

Fig. 3 Scatter plots with LOWESS fits for the local morphometric indices. The measured indices are the number of rods (Nr.Rods), the ratio of the number of rods to the number of plates (Nr.Rods/Nr. Plates), the plate volume density (P1.BV/BV), mean rod slenderness
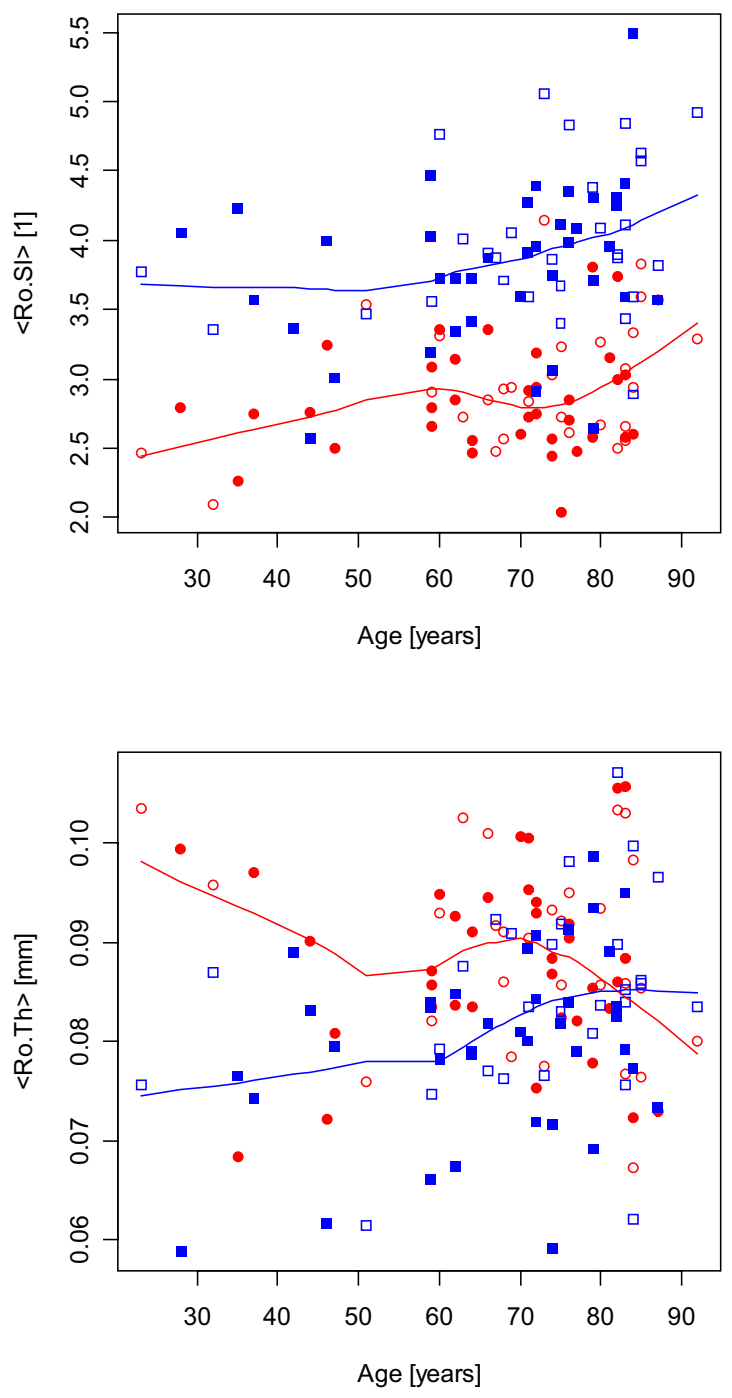

$E$

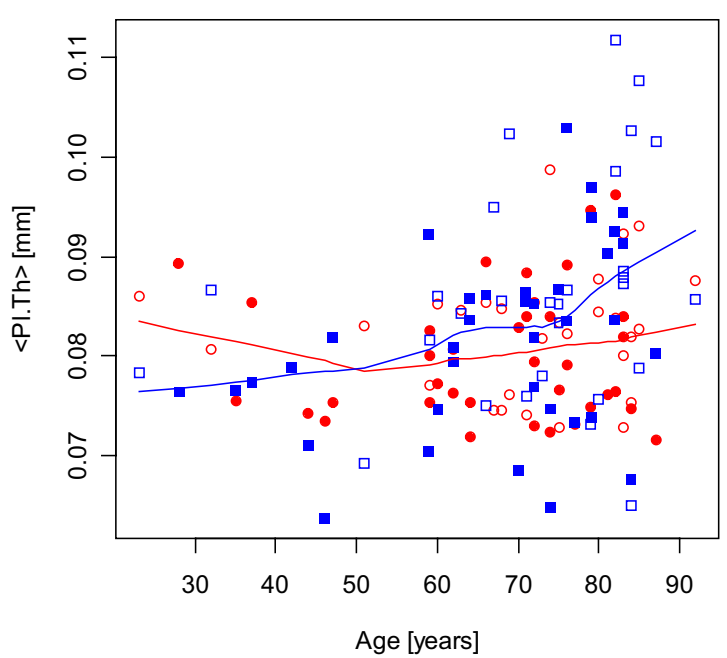

$(<$ Ro.Sl $>)$, mean rod thickness $(<$ Ro.Th $>)$, and mean plate thickness $(<\mathrm{Pl}$.Th $>$ ). Open symbols denote female, and closed symbols denote male, where data from femoral head are displayed as circles $(\circ, \bullet)$ and data from lumbar spine as squares $(\square, \boldsymbol{\square})$ 

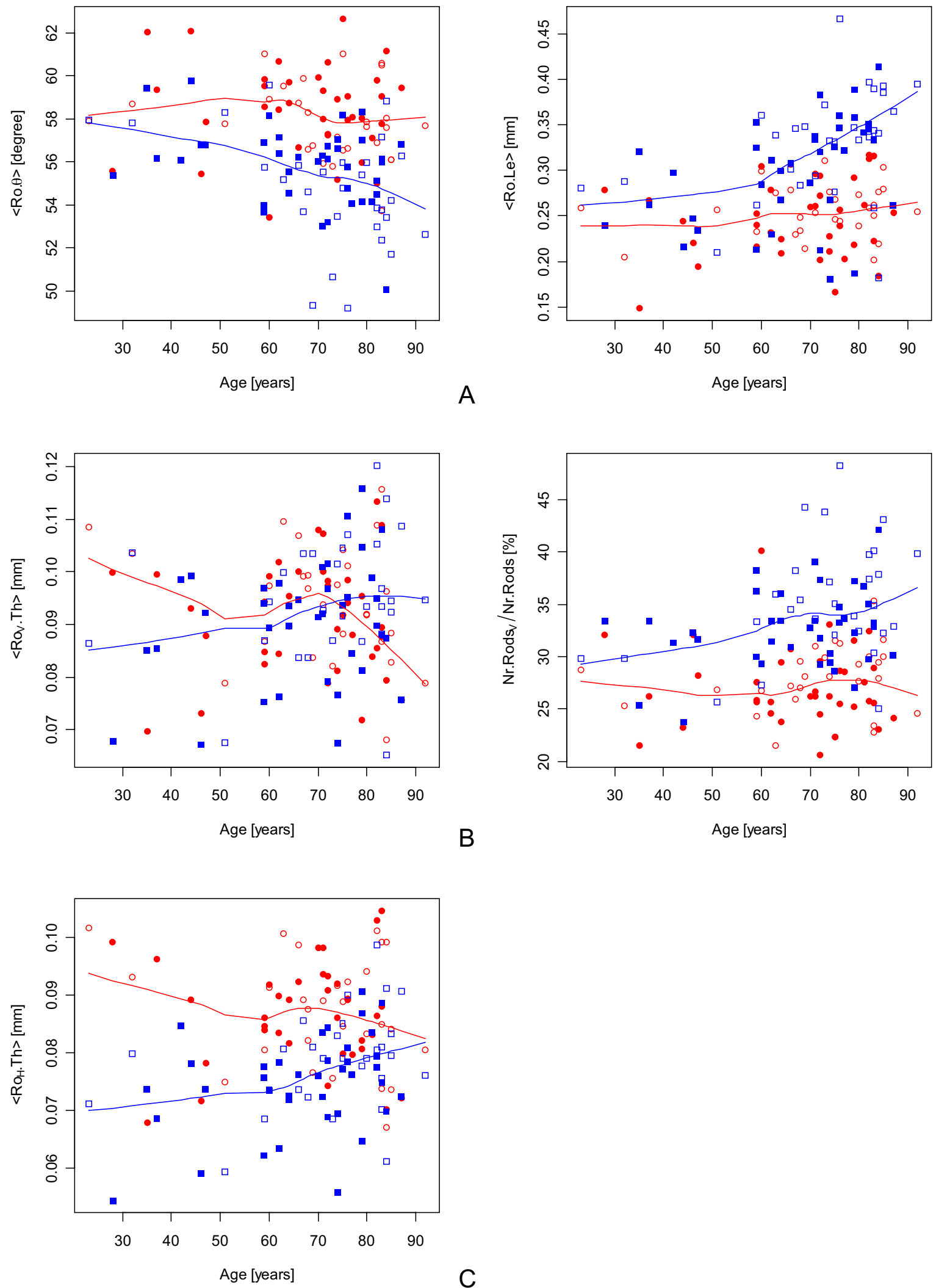

Fig. 4 Scatter plots with LOWESS fits for the local morphometric indices. The measured indices are the mean orientation of rods $(<$ Ro. $\theta>)$, the ratio of the number of vertical rods to the number of rods (Nr.Rods ${ }_{\mathrm{V}} / \mathrm{Nr}$.Rods), the mean thickness of vertical rods $\left(<\mathrm{Ro}_{\mathrm{V}} \mathrm{Th}>\right)$

and the mean thickness of horizontal rods $\left(<\mathrm{Ro}_{\mathrm{H}} . \mathrm{Th}>\right)$. Open symbols denote female, and closed symbols denote male, where data from femoral head are displayed as circles $(\circ, \bullet)$ and data from lumbar spine as squares $(\square, \boldsymbol{\square})$ 
Table $3 P$ values of the analysis of covariance for the local morphometric indices

\begin{tabular}{|c|c|c|c|c|c|}
\hline Index & Site & $\operatorname{Age}^{a}$ & Gender & Age:gender ${ }^{\mathrm{b}}$ & Change $^{c}$ \\
\hline \multirow[t]{2}{*}{ Nr.Rods } & FH & 0.469 & 0.356 & 0.952 & - \\
\hline & LS & 0.002 & 0.626 & 0.788 & -7.9 \\
\hline \multirow[t]{2}{*}{ Nr.Rods/ Nr.Plates } & FH & 0.093 & 0.174 & 0.916 & - \\
\hline & LS & 0.235 & 0.786 & 0.072 & - \\
\hline \multirow[t]{2}{*}{ Pl.BV/BV } & FH & 0.155 & 0.104 & 0.991 & - \\
\hline & LS & 0.775 & 0.318 & 0.379 & - \\
\hline \multirow[t]{2}{*}{$<$ Ro.Sl $>$} & FH & 0.019 & 0.445 & 0.455 & 4.2 \\
\hline & LS & 0.019 & 0.336 & 0.987 & 2.7 \\
\hline \multirow[t]{2}{*}{$<$ Ro.Le $>$} & FH & 0.151 & 0.464 & 0.719 & - \\
\hline & LS & $<0.001$ & 0.122 & 0.973 & 5.7 \\
\hline \multirow[t]{2}{*}{$<$ Ro.Th $>$} & FH & 0.384 & 0.562 & 0.199 & - \\
\hline & LS & 0.003 & 0.147 & 0.894 & 2.6 \\
\hline \multirow[t]{2}{*}{$<\mathrm{Pl}$.Th $>$} & FH & 0.539 & 0.170 & 0.940 & - \\
\hline & LS & 0.006 & 0.166 & 0.960 & 2.6 \\
\hline \multirow[t]{2}{*}{$<$ Ro. $\theta>$} & FH & 0.314 & 0.254 & 0.571 & - \\
\hline & LS & 0.001 & 0.096 & 0.371 & -1.2 \\
\hline \multirow[t]{2}{*}{ Nr.Rods $_{V} /$ Nr.Rods } & FH & 0.731 & 0.559 & 0.363 & - \\
\hline & LS & 0.004 & 0.054 & 0.393 & 4.2 \\
\hline \multirow[t]{2}{*}{$<\mathrm{Ro}_{\mathrm{V}} \cdot \mathrm{Th}>$} & FH & 0.444 & 0.285 & 0.133 & - \\
\hline & LS & 0.031 & 0.246 & 0.786 & 1.8 \\
\hline \multirow[t]{2}{*}{$<\mathrm{Ro}_{\mathrm{H}} \cdot \mathrm{Th}>$} & FH & 0.391 & 0.087 & 0.336 & - \\
\hline & LS & 0.005 & 0.121 & 0.927 & 2.5 \\
\hline
\end{tabular}

$P l . B V / B V$ plate volume density, $<R o . L e>$ mean rod length, $<R o . \theta>$ mean rod orientation, $<R o$.Th $>$ mean thickness of all rods, $<P l . T h\rangle$ mean thickness of all plates, $N r \cdot R o d s_{\mathrm{V}} / \mathrm{Nr}$.Rods ratio of number of vertical rods to number of rods, $<R o_{\mathrm{v}} T h>$ mean thickness of all vertical rods, $<R o_{\mathrm{H}}$. Th $>$ mean thickness of all horizontal rods, $<$ Ro.Sl $>$ mean slenderness of rods, $F H$ femoral head, $L S$ lumbar spine

${ }^{a}$ Bold numbers are smaller than 0.05

${ }^{\mathrm{b}}$ Interaction term age:gender

${ }^{\mathrm{c}}$ Change per decade in percent

\section{Discussion}

This is the first study on age-related changes of 3-D local morphometric indices on human cancellous bone. Only recently a new method for the volumetric spatial decomposition of trabecular bone structures was proposed, enabling local morphometry as applied on individual rods and plates [44-46]. Here we related these new indices to age, separately for men and women, in two different sites (FH and LS). These sites were chosen since they represent platelike and rodlike bone structures. Additionally, to draw a picture of age-related bone changes, we also computed 3-D global morphometric indices. Age-related changes of 3-D global morphometric parameters have already been investigated for tibial cancellous bone [42, 60]. However, we found no study in which global morphometry derived from micro-CT data was related to age in FH and LS. The aim of this study was to achieve a better understanding of age-related bone changes in the trabecular bone structure.

A separate analysis of bone samples from donors aged 59 years and older showed similar trends in all morpho- metric indices as the analysis of the whole data set. However, due to the large variation at these ages, none of the linear regressions were any longer significant. This could indicate that age-related changes are very individual at a given age but tend to be similar if compared over the whole lifetime. For this reason, in this study, only age-related changes over the whole lifetime were considered. The first and most accepted age-related change in trabecular bone structure is bone loss as expressed in a decrease of calcified bone tissue. It is well accepted that such bone loss is an important factor leading to enhanced bone fragility and fracture risk in the elderly. Such bone loss has been demonstrated in many studies for different anatomical sites $[1-3,8-13,16,17,26,42,61,62]$. The results from our study are in line with these findings, and we could demonstrate a linear relationship with age in LS. According to our data, a 90-year-old person showed a decrease in BV/ TV of $38 \%$ in LS as compared with a 30 -year-old subject. In $\mathrm{FH}$, we found no age-related changes although the LOWESS fit in Fig. 2a tends to decrease at higher ages ( $>70$ years). It is a general finding of this study that agerelated changes are more clearly expressed in LS than in FH in all morphometric parameters. It is, of course, important to note that such age-related changes can only apply to an "average" population. Typical for cross-sectional studies, subject variation is too large to make statements about individual time courses.

Similar to BV/TV, BS/TV in LS also decreased with age (6.8\% per decade) in a linear fashion (Table 2$)$. This is not surprising since larger volumes also have larger surfaces, assuming a constant structure shape. The decrease was especially pronounced after the age of 70 (Fig. 2b). Opposed to this, the $\mathrm{BS} / \mathrm{BV}$, a measure for the relative amount of bone surface per bone volume, remained constant throughout life in both sites (Fig. 2c, Table 2), a result supported by earlier findings from quantitative histology [15].

$\mathrm{Tb} . \mathrm{Sp}, \mathrm{Tb} . \mathrm{N}$, and Tb.Th were investigated intensively with many different approaches. Concerning Tb.Sp, there is little controversy, and to our knowledge, all published results indicate an age-related increase $[12,22,23,25-27$, 29,30], which is in line with our findings (Fig. 2d, Table 2). Opposed to Tb.Sp, Tb.Th is of higher controversy and has thoroughly been investigated by means of two-dimensional (2-D) and 3-D methods. In some studies, it was found that the mean $\mathrm{Tb}$.Th decreased significantly with age either in all trabeculae [28-30] or in horizontal trabeculae only $[25,27]$ whereas in other studies, no significant decrease could be observed $[12,22]$. Other studies even speculated that vertical elements that are subjected to compression could increase in thickness as a compensatory response to loss of horizontal trabeculae $[24,63]$.

In our study, we approached the thickness of the trabeculae on a 3-D global as well as on a 3-D true local level. Globally, we found no age-related changes in the $\mathrm{Tb}$. Th (Fig. 2f, Table 2). On a local basis, we investigated the thickness separately for plates and rods, which both showed an increase at higher ages in LS. This increase was found in both vertical and horizontal elements (Fig. 4b,c, Table 3). There are two interpretations to this result: first, trabeculae 
become thicker; second, smaller trabeculae vanish. Since we also found in our study that the number of rods decreases with age, we speculate that the second interpretation is more realistic and that at higher ages, trabeculae first become thinner and finally vanish from the structure.

Increased Tb.Sp implies a decrease in Tb.N, which was found in earlier studies where shortening or complete loss of trabeculae was documented $[18,28]$. In another study, it was found that the Tb.N decreased significantly with age for both horizontal and vertical trabeculae [27]. In our study, we could demonstrate a loss of trabeculae with global and local morphometry. From global morphometry, we found a general loss of Tb.N in LS. From local morphometry, we found a decrease in the number of rods (Nr.Rods) in LS only whereas FH showed no age-related changes. For the LS, this change is very obvious. The structures from this site are already in young people extremely rodlike and thus, if some elements are lost with age, the number of rods decreases as well. In FH, on the other hand, the number of elements is more difficult to interpret. In aging, bones plates eventually get perforated, which results in new, smaller plates and later in new rods. Thus, in FH, the number of elements increases due to plate perforation at the same time as some other elements vanish due to the aging process. Although bone remodeling mechanisms on a cellular level are most likely the same at both sites, they are expressed differently in the remodeling of the structure due to the different initial structure types. This result is corroborated by a recent simulation study [64] where it was demonstrated that the relative amount of bone loss in any given structure was highly and directly correlated to the $\mathrm{BS} / \mathrm{BV}$, a measure for the structure shape.

It was suggested in different studies that buckling is an important failure mode [65-70]. The relative importance of this failure mode clearly increases with increasing slenderness of trabeculae. In our study, we found an increase in the $<$ Ro.Sl>, which was linear in LS and FH. This result is caused by a large increase in $<$ Ro.Le $>$, which was especially pronounced in LS.

In our study, we could demonstrate that local morphometry used in combination with global morphometry may help to improve the understanding of age-related changes in trabecular bone microstructure. However, this study is limited due to the cross-sectional nature and the limited number of samples per age group, resulting in a huge variation in morphometric parameters at any age. In cross-sectional studies, generally, thousands of samples are needed to demonstrate highly significant regressions, wherefore this study only may be considered as an illustration of the potential utility of the technique, highlighting some significant and interesting trends. The high variation in the data may also be part of the reason why no gender differences could be detected although it is generally accepted that osteoporotic fractures occur with a much higher incidence in women than in men $[49,50]$. However, previous studies investigating trabecular bone also reported almost no gender differences on a microstructural level [26, 42], and it was suggested that not only a greater age-related decrease in volumetric bone mineral density in trabecular and cor- tical bone but also other factors, such as a smaller bone size in women compared with men may explain, in a large part, why fragility fractures are more common in elderly women than in elderly men [62].

We would like to summarize our findings as follows: (1) Age related changes are best seen in LS. The reason for this is the extremely rodlike structure type, which is expressed by a high $\mathrm{BS} / \mathrm{BV}$ that is sensitive to both hormonal and environmental changes. In aging people, thin rods are further thinned and finally vanish from the structure, which causes a transformation of the trabecular bone structure to longer and, on average, thicker rods. Due to loss of interconnecting rods, the remaining trabeculae are more likely to buckle, increasing the potential for catastrophic failure. (2) Due to the high variation in almost all morphometric indices at all ages, it seems difficult to predict only from one site the state of the overall skeleton in cross-sectional studies. (3) Trabecular bone from FH is extremely platelike at all ages. Changes in these structures are expressed by a simultaneous thinning and loss of interconnecting trabeculae and perforation of plates leading to new plates and rods. With these changes, connectivity density can either increase or decrease and is therefore not a representative measure for the state of the bone.

In conclusion, our work demonstrates that 3-D local morphometry in combination with 3-D global morphometry are useful tools for the investigation of changes in trabecular bone microstructures. In our study, these tools were used to improve our understanding of age-related microstructural changes in trabecular bone, but these new tools could in future studies also help to better understand microstructural changes related to disease and treatment in individual rod and plate elements separately.

Acknowledgements This study was partly supported by the SNF Professorship in Bioengineering of the Swiss National Science Foundation (FP 620-58097.99 and PP-104317/1) and the European Union BIOMED I concerted action "Assessment of Bone Quality in Osteoporosis". We would also like to thank Dr. H.R. Roth for statistical advice.

\section{References}

1. Beck JS, Nordin BE (1960) Histological assessment of osteoporosis by iliac crest biopsy. J Pathol Bacteriol 80:391-397

2. Caldwell RA (1962) Observations on the incidence, aetiology, and pathology of senile osteoporosis. J Clin Pathol 15:421-431

3. Lindahl O, Lindgren AG (1962) Grading of osteoporosis in autopsy specimens. A new method. Acta Orthop Scand 32:85100

4. Riggs BL (1991) Overview of osteoporosis. West J Med 154 (1):63-77

5. Consensus development conference (1991) Prophylaxis and treatment of osteoporosis (1991). Am J Med 90(1):107-110

6. Consensus development conference (1993) Diagnosis, prophylaxis, and treatment of osteoporosis. Am J Med 94(6):646-650

7. Consensus Development Conference (2000) Osteoporosis prevention, diagnosis, and therapy. NIH consensus statement 17 (1): $1-45$

8. Trotter M, Broman GE, Peterson RR (1960) Densities of bones of white and Negro skeletons. Am J Orthop 42-A:50-58 
9. Bromley RG, Dockum NL, Arnold JS, Jee WS (1966) Quantitative histological study of human lumbar vertebrae. J Gerontol 21(4):537-543

10. Chalmers J, Weaver JK (1966) Cancellous bone: its strength and changes with aging and an evaluation of some methods for measuring its mineral content. II. An evaluation of some methods for measuring osteoporosis. J Bone Joint Surg Am 48 (2):299-308

11. Dunnill MS, Anderson JA, Whitehead R (1967) Quantitative histological studies on age changes in bone. J Pathol Bacteriol 94(2):275-291

12. Wakamatsu E, Sissons HA (1969) The cancellous bone of the iliac crest. Calcif Tissue Res 4(2):147-61

13. Ellis HA, Peart KM (1972) Quantitative observations on mineralized and non-mineralized bone in the iliac crest. J Clin Pathol 25(4):277-86

14. Arnold JS, Wei LT (1972) Quantitative morphology of vertebral trabecular bone. In: Stover B, Jee WS (eds) Radiobiology of plutonium, JW Press, Salt Lake City, pp 333-354

15. Merz WA, Schenk RK (1970) Quantitative structural analysis of human cancellous bone. Acta Anat (Basel) 75(1):54-66

16. Melsen F, Melsen B, Mosekilde L, Bergmann S (1978) Histomorphometric analysis of normal bone from the iliac crest. Acta Pathol Microbiol Scand, A 86(1):70-81

17. Vedi S, Compston JE, Webb A, Tighe JR (1982) Histomorphometric analysis of bone biopsies from the iliac crest of normal British subjects. Metab Bone Dis Relat Res 4(4):231-236

18. Atkinson PJ (1967) Variation in trabecular structure of vertebrae with age. Calcif Tissue Res 1(1):24-32

19. Carter DR, Hayes WC (1977) The compressive behavior of bone as a two-phase porous structure. J Bone Joint Surg Am 59 (7):954-962

20. Rice JC, Cowin SC, Bowman JA (1988) On the dependence of the elasticity and strength of cancellous bone on apparent density. J Biomech 21(2):155-168

21. Hui SL, Slemenda CW, Johnston CC, Jr (1988) Age and bone mass as predictors of fracture in a prospective study. J Clin Invest 81(6):1804-1809

22. Parfitt AM, Mathews CH, Villanueva AR, Kleerekoper M, Frame B, Rao DS (1983) Relationships between surface, volume, and thickness of iliac trabecular bone in aging and in osteoporosis. Implications for the microanatomic and cellular mechanisms of bone loss. J Clin Invest 72(4):1396-1409

23. Kleerekoper M, Villanueva AR, Stanciu J, Rao DS, Parfitt AM (1985) The role of three-dimensional trabecular microstructure in the pathogenesis of vertebral compression fractures. Calcif Tissue Int 37(6):594-597

24. Parfitt AM (1984) Age-related structural changes in trabecular and cortical bone: cellular mechanisms and biomechanical consequences. Calcif Tissue Int 36 (Suppl 1):S123-8

25. Mosekilde L (1988) Age-related changes in vertebral trabecular bone architecture-assessed by a new method. Bone 9(4):247250

26. Mosekilde L (1989) Sex differences in age-related loss of vertebral trabecular bone mass and structure-biomechanical consequences. Bone 10(6):425-432

27. Thomsen JS, Ebbesen EN, Mosekilde LI (2002) Age-related differences between thinning of horizontal and vertical trabeculae in human lumbar bone as assessed by a new computerized method. Bone 31(1):136-142

28. Bergot C, Laval-Jeantet AM, Preteux F, Meunier A (1988) Measurement of anisotropic vertebral trabecular bone loss during aging by quantitative image analysis. Calcif Tissue Int 43(3):143-149

29. McCalden RW, McGeough JA, Court-Brown CM (1997) Agerelated changes in the compressive strength of cancellous bone. The relative importance of changes in density and trabecular architecture. J Bone Joint Surg Am 79(3):421-427

30. Weinstein RS, Hutson MS (1987) Decreased trabecular width and increased trabecular spacing contribute to bone loss with aging. Bone 8(3):137-142
31. Rüegsegger P, Koller B, Müller R (1996) A microtomographic system for the nondestructive evaluation of bone architecture. Calcif Tissue Int 58(1):24-29

32. Müller R, Hildebrand T, Rüegsegger P (1994) Non-invasive bone biopsy: a new method to analyse and display the threedimensional structure of trabecular bone. Phys Med Biol 39 (1):145-164

33. Müller R (2002) The Zürich experience: one decade of threedimensional high-resolution computed tomography. Top Magn Reson Imaging 13(5):307-322

34. Wehrli FW, Saha PK, Gomberg BR, Song HK, Snyder PJ, Benito M, Wright A, Weening R (2002) Role of magnetic resonance for assessing structure and function of trabecular bone. Top Magn Reson Imaging 13(5):335-355

35. Majumdar S (1998) A review of magnetic resonance (MR) imaging of trabecular bone micro-architecture: contribution to the prediction of biomechanical properties and fracture prevalence. Technol Health Care 6(5-6):321-327

36. Majumdar S (2002) Magnetic resonance imaging of trabecular bone structure. Top Magn Reson Imaging 13(5):323-334

37. Hildebrand T, Rüegsegger P (1997) A new method for the model-independent assessment of thickness in three-dimensional images. J Microsc 185:67-75

38. Hildebrand T, Rüegsegger P (1997) Quantification of bone microarchitecture with the structure model index. Comput Methods Biomech Biomed Eng 1(1):15-23

39. Jinnai H, Watashiba H, Kajihara T, Nishikawa Y, Takahashi M Ito M (2002) Surface curvatures of trabecular bone microarchitecture. Bone 30(1):191-194

40. Odgaard A, Gundersen HJ (1993) Quantification of connectivity in cancellous bone, with special emphasis on 3-D reconstructions. Bone 14(2):173-182

41. Ding M (2000) Age variations in the properties of human tibial trabecular bone and cartilage. Acta Orthop Scand 292:1-45

42. Ding M, Odgaard A, Linde F, Hvid I (2002) Age-related variations in the microstructure of human tibial cancellous bone. J Orthop Res 20(3):615-621

43. Kinney JH, Ladd AJ (1998) The relationship between threedimensional connectivity and the elastic properties of trabecular bone. J Bone Miner Res 13(5):839-845

44. Stauber M, Nazarian A, Müller R (2002) Element based morphometric characterization of trabecular bone. Trans Europ Orthop Res Soc 12, pp O89

45. Stauber M, Rapillard L, Zysset PK, Müller R (2003) Element based morphometry - a new tool for the assessment of mechanostructure relationships in trabecular bone. Trans Orthop Res Soc 28:109 New Orleans

46. Stauber M, Müller R (2006) Volumetric spatial decomposition of trabecular bone into rods and plates - A new method for local bone morphometry. Bone in press

47. Hildebrand T, Laib A, Müller R, Dequeker J, Rüegsegger P (1999) Direct three-dimensional morphometric analysis of human cancellous bone: microstructural data from spine, femur, iliac crest, and calcaneus. J Bone Miner Res 14(7): $1167-1174$

48. Dequeker J (1994) Assessment of quality of bone in osteoporosis-BIOMED I: fundamental study of relevant bone. Clin Rheumatol 13 (Suppl 1):7-12

49. Melton LJ, 3rd (2000) Who has osteoporosis? A conflict between clinical and public health perspectives. J Bone Miner Res 15(12):2309-2314

50. Cummings SR, Melton LJ (2002) Epidemiology and outcomes of osteoporotic fractures. Lancet 359(9319):1761-1767

51. Aerssens J, Boonen S, Joly J, Dequeker J (1997) Variations in trabecular bone composition with anatomical site and age: potential implications for bone quality assessment. J Endocrinol 155(3):411-421

52. Dequeker J, Boonen S, Breemans S (1995) Assessment of quality of bone in osteoporosis: clinical characteristics of patient material. Clin Rheumatol 14:596 
53. Müller R, Rüegsegger P (1997) Micro-tomographic imaging for the nondestructive evaluation of trabecular bone architecture. Stud Health Technol Inform 40:61-79

54. Hahn M, Vogel M, Pompesius-Kempa M, Delling G (1992) Trabecular bone pattern factor-a new parameter for simple quantification of bone microarchitecture. Bone 13(4):327-330

55. Bernard TM, Manzanera A (1999) Improved Low Complexity Fully Parallel Thinning Algorithm 10th International Conference on Image Analysis and Processing (ICIAP '99), Venice, Italy

56. Manzanera A, Bernard TM, Prêteux F, Longuet B (1999) Medial faces from a concise 3D thinning algorithm International Conference on Computer Vision (ICCV '99), Kerkyra, Greece, pp 337-343

57. Saha PK, Chaudhuri BB (1996) 3D digital topology under binary transformation with applications. Comput Vis Image Underst 63(3):418-429

58. Cleveland WS (1979) Robust locally weighted regression and smoothing scatterplots. J Am Stat Assoc 74(368):829-836

59. Cleveland WS (1981) Lowess - a program for smoothing scatterplots by robust locally weighted regression. Am Stat 35 (1):54-54

60. Ding M, Hvid I (2000) Quantification of age-related changes in the structure model type and trabecular thickness of human tibial cancellous bone. Bone 26(3):291-295

61. Cummings SR, Black DM, Nevitt MC, Browner WS, Cauley JA, Genant HK, Mascioli SR, Scott JC, Seeley DG, Steiger P, et al. (1990) Appendicular bone density and age predict hip fracture in women. The Study of Osteoporotic Fractures Research Group. JAMA 263(5):665-668
62. Riggs BL, Melton Iii LJ, Robb RA, Camp JJ, Atkinson EJ, Peterson JM, Rouleau PA, McCollough CH, Bouxsein ML, Khosla S (2004) Population-based study of age and sex differences in bone volumetric density, size, geometry, and structure at different skeletal sites. J Bone Miner Res 19(12):19451954

63. Parfitt AM (1989) Plasma calcium control at quiescent bone surfaces: a new approach to the homeostatic function of bone lining cells. Bone 10(2):87-88

64. Müller R (2005) Long-term prediction of three-dimensional bone architecture in simulations of pre-, peri- and post-menopausal microstructural bone remodeling. Osteoporos Int 16 (Suppl 2):S25-S35

65. Hayes WC, Carter DR (1976) Postyield behavior of subchondral trabecular bone. J Biomed Mater Res 10(4):537-544

66. Turner CH (1989) Yield behavior of bovine cancellous bone. J Biomech Eng 111(3):256-260

67. Michel MC, Guo XD, Gibson LJ, McMahon TA, Hayes WC (1993) Compressive fatigue behavior of bovine trabecular bone. J Biomech 26(4-5):453-463

68. Müller R, Gerber SC, Hayes WC (1998) Micro-compression: a novel technique for the nondestructive assessment of local bone failure. Technol Health Care 6(5-6):433-444

69. Nazarian A, Müller R (2004) Time-lapsed microstructural imaging of bone failure behavior. J Biomech 37(1):55-65

70. Gibson LJ (2005) Biomechanics of cellular solids. J Biomech 38(3):377-399 